Published in "Macromolecular Symposia 291-292(1): 75-83, 2010"

which should be cited to refer to this work.

\title{
Coordination Polymer Networks: An Alternative to Classical Polymers?
}

\begin{abstract}
Katharina M. Fromm, ${ }^{* 1}$ Jorge L. Sague,, 2 Laurent Mirolo ${ }^{1}$
Summary: This chapter provides a brief overview on metal ion containing coordination polymer networks, how and why they are made, as an alternative to classical polymers. Our focus will be polymorphism, supramolecular isomerism and pseudopolymorphism. Examples from our group of metal ion containing coordination polymer networks with flexible molecules as building blocks will be highlighted, as they might show parallels with purely organic polymer compounds.
\end{abstract}

Keywords: coordination polymer networks; isomerism; polymorphism; pseudopolymorphism; $x$-ray structure

\section{Introduction}

The term "coordination polymer" was first used by J. C. Bailar in 1964, when he compared organic polymers with inorganic compounds which can be considered as polymeric species. In comparison, he established rules for the building and the required properties of new species involving metal ions and organic ligands. ${ }^{[1]}$

Polymers are defined as high molecular weight molecules formed by the repetition of monomeric units linked with covalent bonds. In comparison, coordination polymers are infinite systems built up with metal ions and organic ligands as main elementary units linked via coordinative and other weak chemical bonds. These compounds are also named metal-organic coordination networks or metal-organic frameworks (MOF), about which recent review articles give a more detailed insight. ${ }^{[2-4]}$

A certain number of principal differences between MOFs and polymers exist

\footnotetext{
${ }^{1}$ Department of Chemistry, University of Fribourg, Chemin du Musée 9, 1700 Fribourg, Switzerland E-mail: katharina.fromm@unifr.ch

2 Department of Chemistry, University of Geneva, 30, Quai E. Ansermet, 1204 Geneva, Switzerland United Kingdom
}

and need to be made clear in order to avoid confusion:

1) Whereas polymers usually show a certain size distribution due to varying chain lengths, MOFs, usually characterized in the solid state, are infinite networks in one, two or three dimensions of space.

2) A polymer is formed by connecting monomer units via covalent bonds, while a coordination polymer network is based mainly on coordinative bonds. Coordinative bonds are formed in equilibrium which may be more on the side of the product and less of the starting materials. For both, weak intermolecular interactions may play an important role on the overall arrangement and influence the properties.

3) As a consequence, in solution, polymers may be identified by their chain length, while for MOFs, the degree of polymerization or oligomerization depends, for instance, on the solvent, the temperature, the pressure etc., i.e. factors which influence the equilibrium.

4) While polymers, due to the connection of a large number of monomers, may possess properties which lead to elastomers, duroplasts, or thermoplasts, the coordination polymer networks are 
usually solid state materials and crystalline, and, therefore, well-ordered in the long range. The latter leads to the fact that the structure of MOFs is usually well-known. The following figures present the structures of such polymers as derived, for most of them, from single crystal x-ray data. That is why the field dealing with coordination polymers is usually called "crystal engineering".

For the chemist, polymeric coordination networks syntheses could be considered as "construction games": the final architecture depends on the building modules (organic ligands, metal ions, their counter ions, solvent molecules) and their compatibilities. In appropriate circumstances, crystals can be considered as the sum of a series of reproducible molecular recognition events. This means that control of the overall arrangement of the modules can be conceivable with prediction of topology and dimensionality, but not of the exact crystal structure (cell parameters). This approach can be compared to supramolecular chemistry and the self-assembly feature, if crystals are regarded as single chemical entities. ${ }^{[5]}$ Self-assembly is based on complementary and explicit interactions between the building blocks in order to generate the final product. ${ }^{[6]}$

The numerous literature contributions in the field of coordination polymers are due to several points, e.g. the incorporation of metal ions in supramolecular networks allows controlled positioning of the metal atom in the final material, and adds properties to the material which are not only based on the metal ions alone, but also on the interplay between the main binding partners, ligands ("linkers") and ions (nodes). Types of metal ions and the distances between them can be chosen so that stable functional solid materials can be tuned. Instead of individual metal ions, clusters can also be used as nodes. Furthermore, the variety of "nodes and linkers" offers to the chemist an infinite number of possibilities for building new species with intriguing architectures, topologies and properties. Moreover, the studies of crystals have become much easier and faster because of the technologic improvements in the field of X-ray measurements and computational resolution techniques.

The bonding energies involved in coordination polymer networks can vary from very strong to very weak. While a coordinative bond can be as strong as a covalent bond in certain cases $\left(c a .50 \mathrm{~kJ} \mathrm{~mol}^{-1}\right.$ on average, but higher values are known too), a group of weaker bonds also contributes to the finally built structure. Among them, hydrogen bonds ${ }^{[9-12]}$ vary from $c a .15$ to $40 \mathrm{~kJ} \mathrm{~mol}^{-1}$ (moderate strength), while $\pi-\pi$ interactions $^{[13]}$ are estimated at $5-10 \mathrm{~kJ} \mathrm{~mol}^{-1}$. Metal-metal interactions based on $d^{10}$ metal cations are controversially discussed, the energy of these bonds being roughly estimated to $c a .5 \mathrm{~kJ} \mathrm{~mol}^{-1}$ for a silver-silver interaction. ${ }^{[14]}$ Metal-aromatic interactions can be formed when metal cations accept $\pi$-electrons from unsaturated organic molecules. The energy of metal-aromatic interactions $^{[8]}$ is not well-known, but evaluated around $5-10 \mathrm{~kJ} \mathrm{~mol}^{-1}$.

Four main synthetic strategies for obtaining coordination polymers are known from the literature, ${ }^{[5,15]}$ many of which also apply for the preparation of all metal-organic compounds. It is important to remember that different processes with the same starting materials may lead to different products: isomeric or polymorphic species which will be discussed later.

First, self-assembly occurs when the reagents are mixed together. Molecular recognition permits the construction of products following pre-determined rules. This technique needs favorable conditions. One of them is the crystal growth in saturated solutions. Ideal concentrations can be achieved by slow evaporation of the mother liquor. Furthermore, the solubility increases with temperature, and crystals can appear during the cooling step, which has to be well-controlled as far as cooling speed and final temperature are concerned. Secondly, diffusion methods may be preferred to get single crystals suitable for $\mathrm{X}$-ray diffraction analysis instead of non- or 
poly-crystalline products, especially if the products are poorly soluble. The principle of this method is to slowly bring into contact the different species. This can happen via the solvent liquid diffusion in which layers are formed; one of them contains the product in an adequate solvent, another is the precipitant solvent and both are separated by a solvent layer. The precipitant solvent slowly diffuses into the separate layer and crystal growth occurs at the interface. The other approach, still concerning diffusion of compounds in solution, is the slow diffusion of reactants. This technique is similar to the one before, the only difference being that the reactants are each dissolved separately in one of the two solutions; the separation between both solutions can be a solution layer and/or physical barriers. Instead of liquid solvents, gels are also used as diffusion and crystallization media in some cases, especially to slow down diffusion and to avoid precipitation of bulk material. Thirdly, hydro(solvo)thermal methods have been recently adopted also for the formation of coordination polymers. Running temperatures range usually between 120 and $260{ }^{\circ} \mathrm{C}$ inside a closed space (autoclave) under autogenous pressure. Under these conditions, the reduced viscosity of water enhances the diffusion process and thus extraction of solids and crystal growth from solution is favored. As the difference of solubility between organic and inorganic components in the same solvent is often a barrier in the formation of single crystals, hydrothermal experiments can be a good alternative to increase the solubility of the starting materials. This crystallization technique is a nonequilibrium synthesis and may lead to metastable products, influenced mainly by the cooling speed at the end of the reaction. Finally, microwave and ultrasonic methods are far less used methods for the coordination polymer formation. These methods are also based on the improvement of solubility in order to better react or crystallize the involved species and products. They remain to be exploited more efficiently.
There are four different kinds of building bricks used for the construction of infinite metal-organic frameworks, crucially influencing the final properties of the compound: ligands, metal ions or clusters, anions and, sometimes, solvent molecules.

The organic ligands act as bridging organic groups between the metal ions. For possible infinite expansion, ligand molecules have to be multidentate with at least two donor atoms, mostly $\mathrm{N}-$, O- or Sdonors. Ligand molecules may differ from each other in their charges: most used ligands are neutral or anionic. Another structure-determining factor is the "body" of the organic ligands: their shapes (rigid or not); their lengths (distance between the coordination functions); their functionalities (further presence of heteroatoms, aromatic rings, alkyl chains etc). And, finally, the ligand molecules can be symmetric, chiral or not, i.e. combining different functionalities in the same molecule. The metal ions or clusters are involved in the structure depending on their size, hardness/softness, ligand-field stabilization energy and coordination geometries (linear, trigonal-planar, T-shaped, tetrahedral, square-planar, square-pyramidal, octahedral, trigonal-prismatic, pentagonal-bipyramidal or trigonal-bipyramidal). In order to counterbalance the positive charges obtained by ligation of neutral ligands to metal ions, counter ions need to be present in the structure. They can influence the metal ion environment (more or less coordinating counter ions) but also the overall structure, being involved in weak interactions or acting as guest molecules in void spaces in the solid state. Finally, solvent molecules may co-crystallize, filling space as guest molecules and/or increasing the number of possible weak interactions in the final solid state packing. They may play a crucial role when it comes to the construction of highly porous materials as reversible guest molecules.

The organization of the building blocks together can lead to MOFs of different dimensionalities: one-, two-, or three-dimensional architectures (Figure 1). 
Metal ions

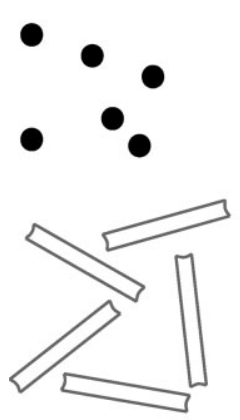

Organic ligands

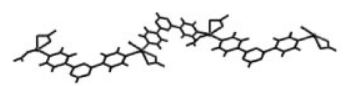

1D

110

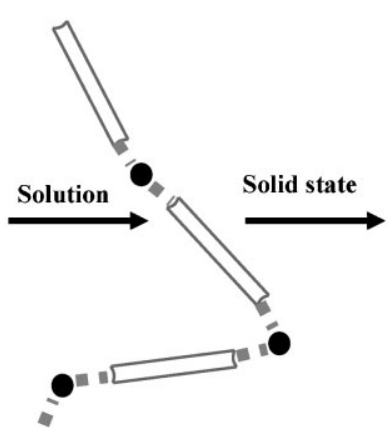

Figure 1.

Formation of coordination polymers. ${ }^{[7,8]}$

Dimensionality is often determined by the nodes (metal ions or clusters): in onedimensional (1D) motifs the metal ion is coordinated with two ligand molecules, metal ions and organic ligands alternate "infinitely", leading to a chain; two-dimensional (2D) compounds are obtained with three or four ligand molecules coordinating around the metal ion and the elementary motif expands now in two directions; with metal ions of higher coordination number (tetrahedral or octahedral nodes), threedimensional (3D) structures can be built.

Obviously, the elementary units are not always so simple and there are a lot of one-, two-, or three-dimensional architectural types depending on the building blocks and the experimental conditions. ${ }^{[5]}$ One other important feature concerning the analyses of coordination polymer architectures is the interpenetration, in which two- or three-dimensional motifs are interweaving. ${ }^{[16]}$

Considering the huge choice of possible building units, one can easily imagine the diversity of new synthesizable materials. In order to illustrate the wide diversity of related coordination polymers, some examples of MOFs will be presented; the aim of the following paragraphs is not to make an exhaustive list, but only to present an overview of the coordination polymer features. They are classified along their dimensionalities. The shown motifs are the most typical ones and are based on coordination interactions between ligand molecules and metal ions. It is clear that other interactions play also an important role during the formation of the crystals, but the definition of the final compounds dimensionality is based on the metal complexation. Due to the abundance of known metal-organic systems, we will only report the systems containing one type of ligand and one type of metal ions.

\section{Results and Discussion}

As building blocks, we chose a flexible ligand family Ln (Scheme 1) in which n can take the values 1, 2, 3, etc. Here, an overview on already published structures will be given, pointing out the structural

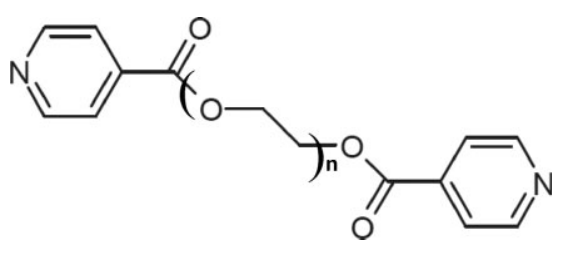

Scheme 1.

Ligand system Ln. 
variety and highlighting their potential for applications.

\section{A Case of Pseudo-Polymorphism}

Pseudo-polymorphism is a term relating to structures which have the same chemical formulae but different number of solvent molecules. Using $\mathrm{CuCl}$ and ligand L1, we obtained two different compounds which indeed differed at first sight only by the content of tetrahydrofuran (THF) as co-crystallizing solvent, namely the yellow $[\mathrm{Cu}(\mathrm{L} 1) \mathrm{Cl}], \mathbf{1}$, and the red $[\mathrm{Cu}(\mathrm{L} 1) \mathrm{Cl}](\mathrm{THF})_{0.5}, \mathbf{2}^{[17]}$ Written as such, one might expect that compound $\mathbf{2}$ can be transformed into compound $\mathbf{1}$ by simply heating to set free the solvent molecules. Yet, the transformation is only observed in the mother liquor, and the reason for this becomes clear when looking at the solid state structures of the two compounds (Fig. 2). In compound $\mathbf{1}$, the ligand adopts the anti conformation, while in $\mathbf{2}$, the gauche conformation is observed. The free ligand crystallizes in the anti conformation, but the energy differences between the two conformers of the ligand are minimal, none of them being essentially preferred. Thus, it is not surprising to find two products with different ligand conformations. It also explains why $\mathbf{2}$ cannot be transformed into $\mathbf{1}$ by simple heating, as more severe reorganizations are necessary. Instead, we propose a solvent-assisted ring opening polymerization mechanism to understand the transformation.

More generally, pseudo-polymorphism is observed quite frequently in coordination chemistry. Compounds containing solvent molecules are also called "solvates" compared to the unsolvated (= solvent-free) compounds.

\section{Polymorphism and Isomerism}

In contrast to pseudo-polymorphism, polymorphism means a) identical chemical formula and b) identical connectivity of atoms within two compounds. ${ }^{[18]}$ Despite the same connectivity, structures may differ from one another. This is an important issue in pharmaceuticals, and some examples will be highlighted here in the case of our ligand systems Ln with $\mathrm{n}=1$ and 2 , and silver salts.

The first example deals with a number of first-order 1D-coordination polymers obtained with $\mathrm{AgNO}_{3}$ and L1. ${ }^{[19]}$ Two

a)

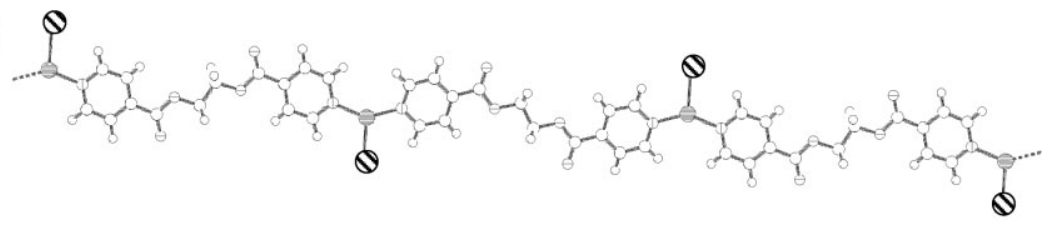

b)

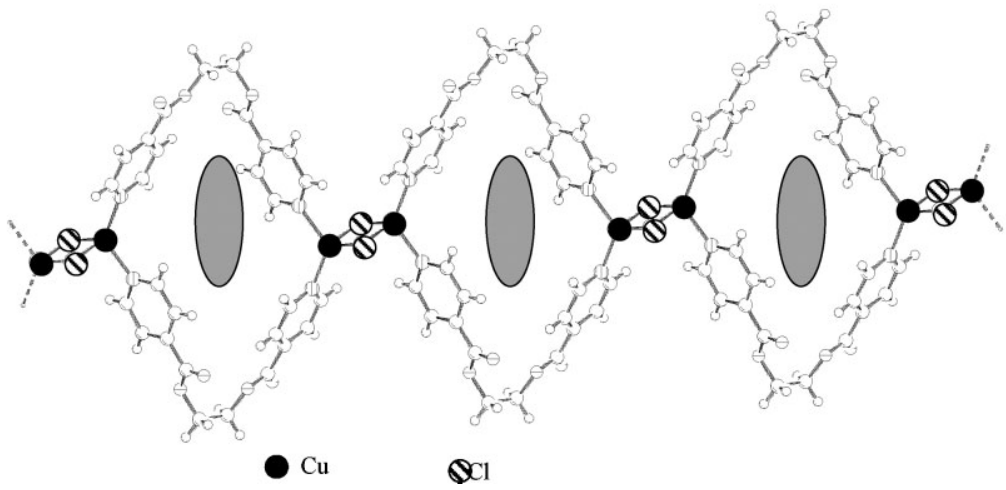

Figure 2.

a) Linear $1 \mathrm{D}$-chains of compound $\mathbf{1}$ with ligand $\mathrm{L} 1$ in the anti conformation; b) Linked loops of the $1 \mathrm{D}$-structure of compound $\mathbf{2}$ with the ligand in the gauche conformation; The THF-molecules are found approximately inside the large rings $\mathrm{Cu}(\mathrm{LI})_{2} \mathrm{Cu}$, as indicated by the grey ovals. 
polymorphs $\left[\mathrm{Ag}(\mathrm{L} 1) \mathrm{NO}_{3}\right], \mathbf{3}$ and $\mathbf{4}$, with ligand L1 in anti-conformation are obtained, in which ligand-metal ion-chains are formed. These are then bridged via interactions with the anions and $\mathrm{H}$-bonding into $2 \mathrm{D}$-structures. The connectivity in the so-obtained structures is identical, but the layout of the ligands with respect to each other is different (Fig. 3).

While compounds 3 and $\mathbf{4}$ are real polymorphs, a third structures exists, still having the same chemical formula, namely $\left[\mathrm{Ag}(\mathrm{L} 1) \mathrm{NO}_{3}\right], \mathbf{5}$, but in which the connectivity between the ions and ligands is different than in $\mathbf{3}$ and $\mathbf{4}$. As shown in Figure 4, the compound forms double chains, running parallel to each other and linked via metal-metal interactions.

This case, i.e. having the same formula but different spatial connectivity, is called isomerism. To this isomer $\mathbf{5}$, a solvate (or pseudo-polymorph) exists, where two water molecules co-crystallize in the solid state. Interestingly, we were able to show the mechanism of water uptake and release in the solid state by the sliding of the doublechains against each other, allowing thus water molecules to "slip in" or "get squeezed out" of the structure. This is reported in detail elsewhere. ${ }^{[19]}$ This claylike behavior is possible due to the similarity of the structure of $\mathbf{5}$ with its solvate, namely $\left[\mathrm{Ag}(\mathrm{L} 1) \mathrm{NO}_{3}\right]\left(\mathrm{H}_{2} \mathrm{O}\right)_{2}$, compound 6 . The water molecules are "simply" sliding into the structure, and the solid state is swelling upon water uptake, and shrinking when losing the solvent molecules again. In $\mathbf{5}$ as well as $\mathbf{6}$, the double-chains are present, and the ligand adopts the same conformation, namely the anti one.

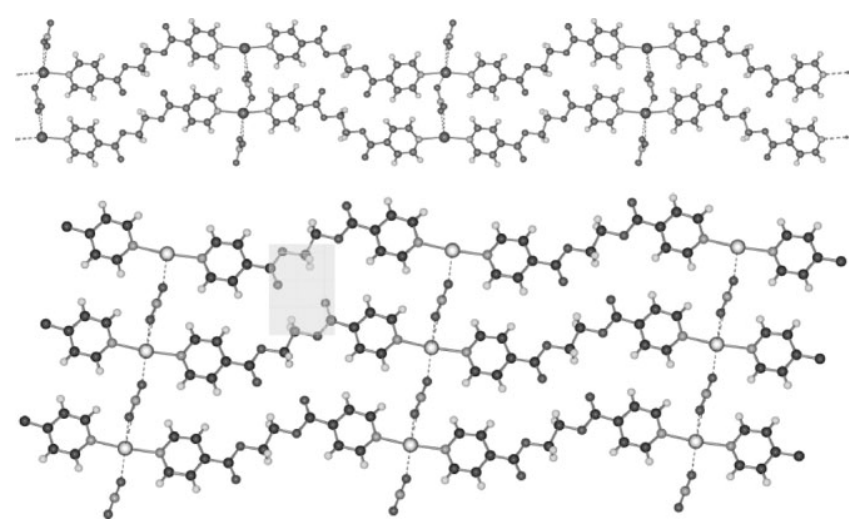

Figure 3.

The two polymorphs 3 and $\mathbf{4}$, exhibiting different solid state symmetry in the ligand arrangements. Blue: Ag-ions, red: oxygen, green: nitrogen, grey: carbon; white: hydrogen.

a)

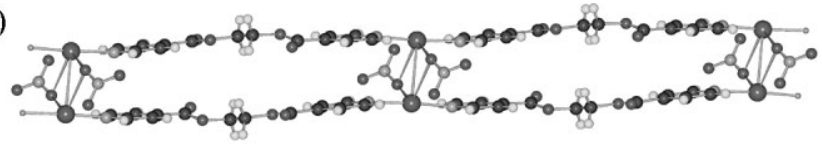

b)

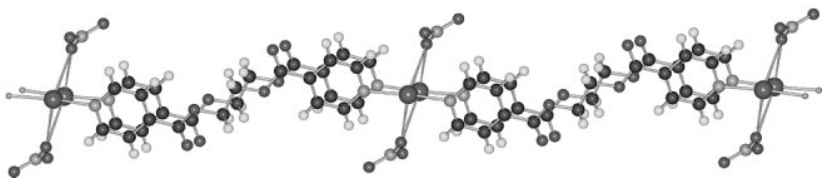

Figure 4.

Double-chains of 5, top (a) and side (b) view. Same color code as in Fig. 3. 
Yet, another solvate, in which the ligand adopts the gauche conformation, and which has only one water molecule, is present in the case of $\left[\mathrm{Ag}(\mathrm{L} 1) \mathrm{NO}_{3}\right]\left(\mathrm{H}_{2} \mathrm{O}\right)$, 7. This is, as for the copper compounds, not directly transformable into $\mathbf{5}$ or $\mathbf{6}$, as the reorganization is too important. Its structure is also based on double-chains, but the gauche conformation of the ligand leads to an undulating structure (Fig. 5). ${ }^{[20]}$

\section{Polymorphism in More Flexible Systems}

The longer the ligand, the more flexible it becomes in principle by the increasing number of single bonds around which it may rotate. Thus, ligand L2 with $\mathrm{n}=2$ possesses one more ethylene oxide unit in the middle spacer. Surprisingly, it adopts by itself an all-gauche conformation in the solid state. ${ }^{[21]}$ This leads again to different possibilities of binding to silver ions. Thus, one possibility is to form molecular entities in which two ligands bind to two silver ions so as to obtain a ring-like structure shown in Figure 5a. Thus, the compound $\left[\mathrm{Ag}_{2}(\mathrm{~L} 2)_{2}\right]\left(\mathrm{ClO}_{4}\right)_{2}, \mathbf{8}$, is obtained forming a molecular structure. To compound $\mathbf{8}$, a polymorph exists, 9, in which the connectivity is identical, but instead of forming a molecular ring structure, the rings open up and connect into a 1D-helical arrangement, as shown in Figure 5b.

With anions other than perchlorate, e.g. the nitrate anion, three metallacyclic isomers are obtained, two of which are

a)

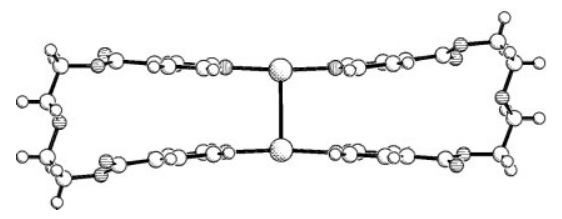

polymorphs, and one is a solvate. The three compounds can thus be written as $\left[\mathrm{Ag}_{2}(\mathrm{~L} 2)_{2}\right]\left(\mathrm{NO}_{3}\right)_{2}\left(\mathrm{H}_{2} \mathrm{O}\right)_{\mathrm{n}}$, for $\mathrm{n}=0$ (compound 10), 0 (polymorph $\mathbf{1 1}$ of 10) or 2 (compound 12). ${ }^{[22]}$

These are results obtained with a metal to ligand ratio of $1: 1$. Changing this ratio by increasing the proportions of ligand to twice that of the metal ions in solution, new compounds may be obtained. This is the case of ligand L2 which reacts with $\mathrm{AgPF}_{6}$ to yield the compound $\left[\mathrm{Ag}(\mathrm{L} 2)_{2}\right]\left(\mathrm{PF}_{6}\right), \mathbf{1 3}^{[23]}$

This is the only structure for which some sort of intercalation of chains and rings is observed, namely a type of polycatenate, linked into two dimensions to yield a "silver chain mail" structure. The structure of this compound is schematized in Figure 6. Indeed, if such an interwoven compound could be made as a classical polymer, very good elastic properties might be expected. The corresponding 1:1 compound $[\mathrm{Ag}(\mathrm{L} 2)]\left(\mathrm{PF}_{6}\right), \mathbf{1 4}$, has a ring structure like compound $\mathbf{8}$, but now with the $\mathrm{PF}_{6}^{-}$-anions bridging the two silver ions.

Even longer ligands Ln with $\mathrm{n}>2$ may lead to molecular ring structures in ratios of ligand to metal ion of $1: 1 .^{[24]}$ In addition to ring structures and depending on the choice of counter ion (coordinating or non-coordinating to silver ions), double-helical 1D-polymers may be obtained. ${ }^{[24]}$

b)

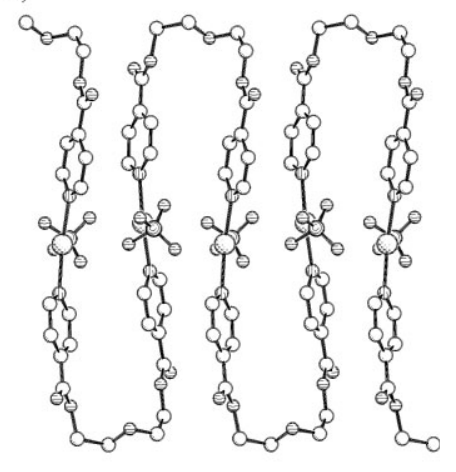

Figure 5.

a) metalla-cycle of compound $\mathbf{8}$, anions omitted for clarity; b) 1D-helix of $\mathbf{9}$, anions shown. 
a)

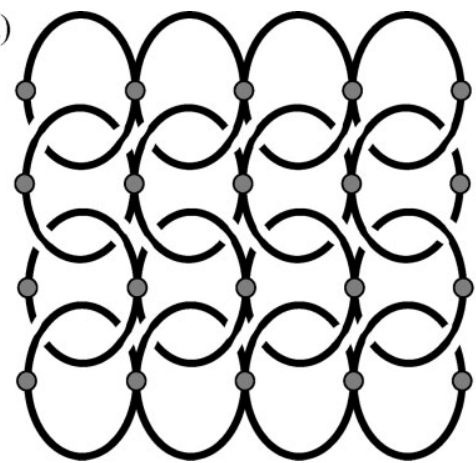

b)

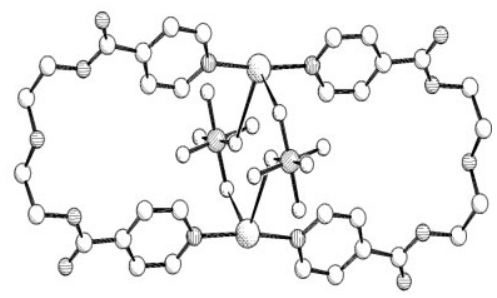

Figure 6.

a) schematic representation of $\mathbf{1 3}$; b) molecular structure of $\mathbf{1 4}$.

The literature gives also such doublehelical, silver-containing chains with the nicotinic derivatives of our ligand system, namely L'4 and L'6, of which the structures are given as examples in Figure $7 .{ }^{[25]}$

In each of these cases, the ligands employed are pure and have a defined length, not a molecular weight distribution as in classical polymers. Thus, well-ordered, crystalline material is obtained, and the structures are well-known and investigated by single-crystal $\mathrm{x}$-ray analysis. The question remains of what kind of systems will be obtained when even longer and more

a)

a)<smiles>O=C(OCCOCCOCCOCCOC(=O)c1cccnc1)c1cccnc1</smiles><smiles>O=C(OCCOCCOCCOCCOCCOCCOC(=O)c1cccnc1)c1cccnc1</smiles>

2 b)

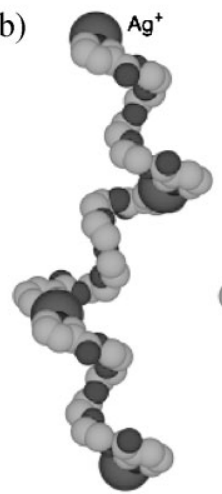

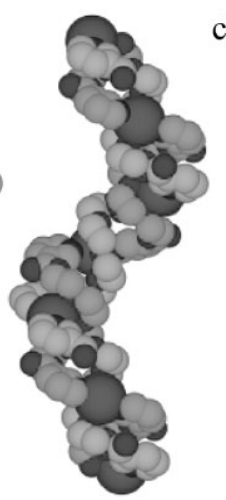

c)

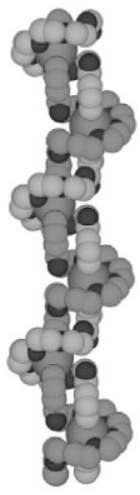

Figure 7.

a) Ligands L'4 and L'6; b) simple polymer chain of L'4 with silver ions (left) and its double-helix (right); c) Doublehelix of L'6 with silver ions. 
flexible ligands are used, e.g. L12 or L20, and also, what a mixture of ligand lengths will lead to upon coordination to metal ions.

We have investigated rather short systems so far, but ligands Ln with $\mathrm{n}>6$ are currently being investigated as ligands for silver ions. We do this also with respect to possible applications in hygienic fields, as silver compounds are generally known to be anti-microbial agents.

\section{Conclusion}

So far, the coordination polymer networks described in the literature are mainly crystalline, or, at least, solid materials. They usually do not possess the properties of many classical polymers, e.g. elasticity, plasticity, etc. However, similar systems with mixed ligand lengths and longer chains might lead to new materials, and future investigations will shed light into this field of coordination chemistry.

Acknowledgements: This work was supported by the Swiss National Science Foundation and the NANO NCCR.

[1] J. C. Bailar, Jr., Prep. Inorg. React. 1964, 1, 1.

[2] A. Y. Robin, K. M. Fromm, Coord. Chem. Rev. 2006, 250, 2127-2157.

[3] K. M. Fromm, Coord. Chem. Rev. 2008, 252(8+9), 856-885.

[4] U. Mueller, J. Mat. Chem. 2006, 16, 626-636; K. Uemura, R. Matsuda, S. Kitagawa, J. Solid State Chem. 2005, 178, 2420-2429; S. Kitagawa, R. Kitaura, S.-I. Noro, Angew. Chem. Int. Ed. 2004, 43, 2334-2375; S. Kitagawa, S. Noro, Comp. Coord. Chem. II 2004, 7,
231-261; B. Kesanli, W. Lin, Coord. Chem. Rev. 2003, 246, 305-326; S. R. Batten, K. S. Murray, Coord. Chem. Rev. 2003, 246, 103-130; J. R. Puddephatt, Coord. Chem. Rev. 2001, 216-217, 313-332; M. J. Zaworotko, Chem. Commun. 2001, 1-9; R. Robson, Dalton Trans. 2000, 3735-3744; M. W. Hosseini, Coord. Chem. Rev. 2003, 240, 157-166.

[5] B. Moulton, M. J. Zaworotko, Chem. Rev. 2001, 101, 1629.

[6] J.-M. Lehn, Supramolecular Chemistry: Concepts and Perspectives, VCH, Weinheim 1995.

[7] A. J. Blake, N. R. Champness, P. Hubberstey, W.-S. Li, M. A. Withersby, M. Schroder, Coord. Chem. Rev. 1999, $183,117$.

[8] S. A. Barnett, N. R. Champness, Coord. Chem. Rev. 2003, 246, 145.

[9] T. Steiner, Angew. Chem. Int. Ed. 2002, 41, 48.

[10] A. Nangia, Cryst. Eng. Commun. 2002, 4, 93.

[11] G. Aullon, D. Bellamy, A. G. Orpen, L. Brammer, E. A. Bruton, Chem. Commun. 1998, 653.

[12] G. R. Desiraju, Acc. Chem. Res. 1996, 29, 441.

[13] C. Janiak, J. Chem. Soc. Dalton Trans. 2000, 3885.

[14] P. Pyykkö, Chem. Rev. 1997, 97, 597.

[15] W. Clegg, Compreh. Coord. Chem. 2004, 1, 579.

[16] L. Carlucci, G. Ciani, D. M. Proserpio, Coord. Chem. Rev. 2003, 246, 247-289.

[17] A. Y. Robin, K. M. Fromm, H. Goesmann, G. Bernardinelli, Cryst. Eng. Commun. 2003, 5, 405-410. [18] D. Braga, F. Grepioni, Chem. Soc. Rev. 2000, 29, 229-238; D. Giron, J. Therm. Anal. \& Calorim. 2001, 64, 37-60; R. J. Sarma, J. B. Baruah, Cryst. Growth \& Des. 2007, 7, 989-1000.

[19] A. Y. Robin, J. L. Sagué Doimeadios, A. Néels, T. Vig Slenters, K. M. Fromm, Inorg. Chim. Acta 2007, 360, 212-220.

[20] A. Y. Robin, J. L. Sagué, K. M. Fromm, Cryst. Eng. Commun. 2006, 8, 403-416.

[21] K. M. Fromm, J. L. Sagué Doimeadios, A. Y. Robin, Chem. Commun. 2005, 36, 4548-4550.

[22] J. L. Sagué, M. Meuwly, K. M. Fromm, Cryst. Eng. Commun. 2008, 10, 1542-1549.

[23] J. L. Sagué, K. M. Fromm, Crys. Growth \& Des. 2006, 6, 1566-1568.

[24] J. L. Sagué, PhD Thesis, University of Basel, 2006. [25] A. Jouaiti, M. W. Hosseini, N. Kyritsakas, Chem. Commun. 2003, 472-473. 